\section{Development of a high-perfor- mance liquid chromatography method to assess bisphenol $F$ levels in milk}

\author{
Serena Santonicola, ${ }^{1}$ Maria Carmela \\ Ferrante, ${ }^{2}$ Giampaolo Colavita, ${ }^{1}$ \\ Raffaelina Mercogliano ${ }^{2}$ \\ ${ }^{1}$ Department of Medicine and Health \\ Sciences, University of Molise, \\ Campobasso; ${ }^{2}$ Department of Veterinary \\ Medicine and Animal Production, \\ University of Naples, Italy
}

\begin{abstract}
Bisphenol $\mathrm{F}$ (BPF) is a bisphenol A (BPA) analogue. As an endocrine disruptor, BPF shows a similar BPA hormonal activity and greater endocrine effects. To assess BPF levels in milk a selective method based on solvent extraction with acetonitrile, solid-phase extraction (SPE), high-performance liquid chromatography with fluorescence detection (HPLC-FD) system, was developed. The method showed high recovery values (from 97.60 to $107.16 \%$ ), and good detection and quantification limits $(\mathrm{LOD}=0.03 \mu \mathrm{g} / \mathrm{L}$; LOQ $=0.1 \mu \mathrm{g} / \mathrm{L})$. To validate the analytical method, quantitative analyses of n.20 milk samples of whole milk were preliminarily carried out applying a monitoring system based on the control of different stages of pasteurized whole milk processing at a dairy company.

The proposed method is simple, sensitive, and might be suitable to detect BPF residues in milk processing. At the dairy company, the occurrence of BPF levels ranging from $<\mathrm{LOQ}$ to $2.956 \mu \mathrm{g} / \mathrm{L}$ was observed. Further analyses and better knowledge about the occurrence, toxicity, and exposure levels of BPF analogue in milk, particularly for vulnerable consumer categories, are needed.
\end{abstract}

\section{Introduction}

Bisphenol F (BPF) (Bis (4-hydroxyphenyl-methane) is a bisphenol A (BPA) (2,2-Bis(4-hydroxyphenyl-propane) analogue that shows a broad range of industrial applications, particularly as food packaging coating, owing to low viscosity and better resistance to solvents (Usman et al., 2019).

As a result of environmental pollution, bioaccumulation, and leaching from packaging materials bisphenols may enter the human food chain producing endocrine effects (Russo et al., 2019). BPF is an endocrine disruptor (ED) and shows a simi- lar BPA hormonal activity and critical endocrine effects on metabolism, growth, reproduction, fetal and sexual development, gender behavior, stress response, and insulin production. In addition, BPF contributes through synergistic effects to obesity and diabetes in childhood and immune system impairment (Bansal et al., 2018; Andújar et al., 2019; Dragone et al., 2020). Restrictions on the use of BPA in certain consumer products have been suggested and a specific migration limit (SML) of $0.05 \mathrm{mg} / \mathrm{kg}$ into/onto food from varnishes or coatings applied to food contact materials was fixed (Regulation (EU) No 2018/213). Structural BPA analogues, such as BPF and bisphenol S (BPS) $\left(4,4^{\prime}\right.$-sulfonyl bisphenol), as replacements of BPA, are gradually entering the food market (Andújar et al., 2019).

Milk is an important source of exposure to EDs, including bisphenols (Santonicola et al., 2018; Lestido-Cardama et al., 2020). BPA and its analogues may enter the milk chain at the farm during milk production, and due to the leaching from plastic parts, thermal treatments, and packaging conditions at the milk dairy processing plant (Casajuana and Lacorte, 2004).

The potential contamination of BPA analogues in the milk chain is a public health concern. A temporary tolerable daily intake (tTDI) of $4 \mu \mathrm{g} / \mathrm{kg}$ body weight/day for only BPA has been fixed (EFSA, 2015). In a previous study a high-performance liquid chromatography (HPLC) method was applied to whole milk samples at different stages of milk processing (raw milk, pasteurization, and milk packaging) to evaluate the BPA exposure in different age category of consumers. Results showed the occurrence of BPA in all considered steps always below the tTDI level (Mercogliano et al., 2021).

Currently, data on the occurrence and human exposure to BPA analogues through food consumption are scarce (Gonzalez et al., 2020). Considering the similarity between BPF and BPA structure and toxicity, experimental studies suggested that BPF might be not a safe alternative, and both bisphenols should be considered for a more comprehensive risk assessment (Andújar et al., 2019).

Based on the previous method developed to determine BPA levels (Mercogliano et al., 2021), the present study aimed to develop and validate a selective HPLC method to assess levels of the analogue BPF in whole milk during milk processing. To improve the analytical method quantitative analyses on milk samples were preliminarily carried out.
Correspondence: Raffaelina Mercogliano, Department of Veterinary Medicine and Animal Production, University of Naples, Via F. Delpino, 1, 80137, Napoli, Italy.

E-mail: raffaella.mercogliano@unina.it

Key words: Bisphenol F, High-performance liquid chromatography method, Milk.

Conflict of interest: The authors declare no potential conflict of interest.

Contributions: The authors contributed equally. Funding: None.

Ethical approval: Not required (the study was carried on samples of commercial milk).

Received for publication: 14 July 2021.

Revision received: 20 October 2021.

Accepted for publication: 26 October 2021.

This work is licensed under a Creative Commons Attribution-NonCommercial 4.0 International License (CC BY-NC 4.0).

(C) Copyright: the Author(s), 2021

Licensee PAGEPress, Italy

Italian Journal of Food Safety 2021; 10:9975 doi:10.4081/ijfs.2021.9975

\section{Materials and methods}

\section{Reagents and standards}

BPF standard (minimum purity of $99 \%$ ) was purchased from Sigma-Aldrich (Poole, UK). Methanol and acetonitrile HPLC grade were provided by Carlo Erba (Milan, Italy). The solid-phase extraction (SPE) cartridges (Chromabond C18, MachereyNagel, Duran, Germany) were purchased from Delchimica (Naples, Italy).

\section{Apparatus}

A Jasco HPLC apparatus equipped with a Jasco quaternary pump 2089 plus combined with a fluorescence detector $821-F p$ (HPLC/FD) (Jasco, Easton, USA) and a Synergy column $4 \mu \mathrm{m}$ Fusion-RP $80 \AA$ (250 by $4.60 \mathrm{~mm}$ inside diameter; Phenomenex, Torrance, CA) were used for the analyses.

The mobile phase was acetonitrilewater $(70: 30, \mathrm{vol} / \mathrm{vol})$ at a flow rate of 0.9 $\mathrm{mL} / \mathrm{min}$ in isocratic mode. The analyses were carried out at room temperature. The fluorescence detector was set at $273 \mathrm{~nm}$ excitation and $300 \mathrm{~nm}$ wavelength emission. BPF was identified based on the retention time.

\section{Quality control/quality assurance}

To validate the method, the quality parameters of linearity, limit of detection 
(LOD) and quantification (LOQ), precision, trueness and selectivity using fortified blank samples were evaluated.

Pasteurized milk in glass bottles obtained from the market, checked not to contain BPF, were used as blank samples. Calibration curve was obtained by external standard calibration method analysis with the blank samples fortified at levels of concentration of BPF standard solutions. LOD and LOQ were calculated based on the signal-to-noise ratio of $3: 1$ and 10:1, respectively.

Precision was evaluated as repeatability (intraday precision) and intermediate precision (interday precision) by spiked blank samples at two concentrations (10.0 and $50.0 \mu \mathrm{g} / \mathrm{L}$ and expressed as a percentage of Relative Standard Deviation (\% RSD). Trueness was evaluated by BPF recovery in spiked blank samples at low $(10.0 \mu \mathrm{g} / \mathrm{L})$ and high concentrations $(50.0 \mu \mathrm{g} / \mathrm{L})$. Selectivity was evaluated by comparing standard and spiked blank samples.

\section{Milk sampling and BPF extraction method}

The whole pasteurized milk chain production at a dairy company was preliminary monitored. Thermal treatment conditions of the milk pasteurization were $71.5^{\circ} \mathrm{C}$ for $15 \mathrm{~s}$. The fat content was $3.4-3.7 \%$ in raw milk, and $3.5 \%$ in pasteurized and cardboard packaged milk. A monitoring system based on the control of each stage of milk processing was applied at the following steps: raw milk from the cooling tank (A), raw milk from the storage tank (B), milk at the end of the pasteurization $(\mathrm{C})$, pasteurized milk from the storage tank (D), and cardboard packaged milk (E). Five milk samples were weekly collected for 4 weeks. A total number of n.20 milk samples was analyzed. A quantity of $1.0 \mathrm{~mL}$ of milk was mixed with $3.0 \mathrm{~mL}$ of deionized water and sonicated for 15 minutes at room temperature in ultrasonic apparatus $(40 \mathrm{kHz}$; Branson ultrasonic 2210, Danbury, CT). Before loading the sample, the SPE cartridge was conditioned with $10.0 \mathrm{~mL}$ of acetonitrile and equilibrated with $10.0 \mathrm{~mL}$ of deionized water. Then the cartridge was washed under vacuum with $8.0 \mathrm{~mL}$ of water and with 14.0 and $16.0 \mathrm{~mL}$ of water and methanol solutions $(80: 20$ and $60: 40$, $\mathrm{vol} / \mathrm{vol})$, respectively.

The analytes were eluted with $10.0 \mathrm{~mL}$ of acetonitrile. The extracts were dried under $\mathrm{N}_{2}$, and then $1.0 \mathrm{~mL}$ of acetonitrile was added. Quantitative analysis was performed by HPLC/FD.

\section{Results}

\section{Linearity, repeatability, detection and quantification limit, recovery, selectivity}

Working standard BPF solutions from 0.1 to $100 \mu \mathrm{g} / \mathrm{L}$ were prepared by diluting aliquots of the stock solution $(100 \mathrm{mg} / \mathrm{L})$ in acetonitrile. A $50 \mu 1$ volume of the standard BPF solution was injected into the HPLC system. The BPF retention time was 3.29 min. Calibration curve for concentrations from 0.1 to $100 \mu \mathrm{g} / \mathrm{L}$ versus detector responses (peak area) was obtained with a linear regression program. The coefficient value $\left(\mathrm{R}^{2}\right)$ of the calibration curve was equal to 0.999 (Figure 1). When BPF was measured consecutively (n. 3 times) in the 10.0 and $50.0 \mu \mathrm{g} / \mathrm{L}$ in fortified blank samples to evaluate the repeatability of the analytical method, the RSD was found to be 4.46 and $1.09 \%$, respectively. Interday precision, as repeatability within-day (n.2), was 14.83 and $5.481 \%$ RSD at high (50.0 $\mu \mathrm{g} / \mathrm{L})$ and low $(10.0 \mu \mathrm{g} / \mathrm{L})$ concentration levels, respectively. The evaluation of instrumental quality parameters resulted in LOD and LOQ values of 0.03 and $0.1 \mu \mathrm{g} / \mathrm{L}$, respectively. BPF recovery rates for milk are 97.60 to $107.16 \%$ at low and high concentration levels. The comparison between standard and blank samples showed the absence of interference peaks.

\section{Application of extraction and analy- sis methods}

Milk samples collected at the stages AB-C-D, and E of milk processing were extracted and analyzed as described above. The results showed the lowest concentrations in raw milk from the storage tank (BPF mean value: $0.268 \mu \mathrm{g} / \mathrm{L}$ ) and the highest in pasteurized milk samples from the storage tank (BPF mean value: 1.211 $\mu \mathrm{g} / \mathrm{L})$ (Table 1).

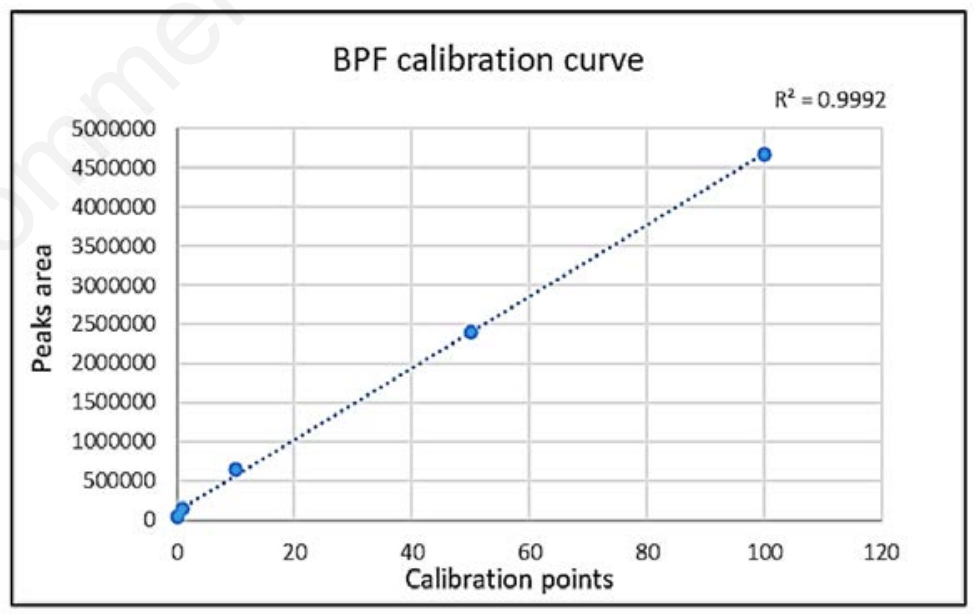

Figure 1. Bisphenol F calibration curve.

Table 1. BPF levels in milk samples collected at different stages of milk processing.

\begin{tabular}{|c|c|c|c|c|c|}
\hline & Milk processing stage & N. samples & Bis & nol F, & \\
\hline & & & Range & mean & median \\
\hline A & Raw milk from the cooling tank & 4 & $0.395-2.956$ & 1.205 & 0.734 \\
\hline B & Raw milk from the storage tank & 4 & $<$ LOQ-0.633 & 0.268 & 0.219 \\
\hline $\mathrm{C}$ & Milk at the end of the pasteurization & 4 & $0.425-0.796$ & 0.541 & 0.472 \\
\hline D & Pasteurized milk from the storage tank & 4 & $0.412-2.686$ & 1.211 & 0.872 \\
\hline $\mathrm{E}$ & Cardboard packaged milk & 4 & $<\mathrm{LOQ}-1.019$ & 0.404 & 0.299 \\
\hline
\end{tabular}

$<$ LOQ (limit of quantification) 


\section{Discussion}

The bisphenol quantification at trace or ultra-trace levels in samples characterized by a complex and variable matrix composition is still challenging. Sample treatment is matrix-dependent and common steps include sample pretreatment, extraction of analytes from the matrix, and cleanup of the extracts to remove interferences (CaballeroCasero et al., 2016; Tuzimski and Szubartowski, 2019). The most critical part of the analysis of milk samples is the cleanup step, because coextracted compounds may inhibit the detection of target compounds through HPLC analyses (Casajuana and Lacorte, 2004). Increasing progress was made during the past years regarding the development of techniques of samples analysis and various methods have been used to extract bisphenols from foodstuff (Tuzimski and Szubartowski, 2019).

In a previous study on the occurrence of BPA in whole milk (Mercogliano et al. 2021), fat was removed from the milk samples using 10.0 and $14.0 \mathrm{~mL}$ of water and methanol solutions (80:20 and 60:40, $\mathrm{vol} / \mathrm{vol})$. To detect BPF levels in milk in the present study fat was removed from the milk samples using a higher volume of water and methanol solutions, and acetonitrile was used as a solvent for the BPF extraction.

The impurities were effectively removed from the samples also through the use of SPE cartridges, and the chromatograms were free of interferences. To detect the BPF, unlike the method of extraction used for BPA levels, milk samples were diluted with water to reduce viscosity and sonicated for emulsion destabilization before the SPE phase. In this way, a better flow rate was achieved during SPE (Caballero-Casero et al., 2016; Mercogliano et al. 2021). Sample treatment resulted in good recovery rates, from 97.60 to $107.16 \%$, which fell into the acceptable range of $70-120 \%$ suggested by the Codex Alimentarius requirement (Hao et al., 2018).

HPLC equipped with a fluorescence detector for the determination of BPA in milk is commonly used because the system is easy to perform, and its sensitivity is high (Kang and Kondo, 2003). Recently, liquid chromatography-tandem mass spectrometry has been widely used because it provides high sensitivity for bisphenol analyses (Caballero-Casero et al., 2016). However, the availability of a validated HPLC-FD method can allow more laboratories to monitor bisphenols in milk without using expensive techniques (Grumetto et al., 2013). According to the literature, the proposed HPLC method to detect BPF levels in milk samples showed good performance (Leepipatpiboon et al., 2005; Hao et al., 2018; Xiao et al., 2020). The achieved LOQ $(0.1 \mu \mathrm{g} / \mathrm{L})$ was lower than the specific migration limit of $0.05 \mathrm{mg} / \mathrm{kg}$ fixed only for BPA (Regulation (EU) No 2018/213), demonstrating the good sensitivity of the method. Excellent linear regression coefficient $(>0.9900)$ was obtained covering a large concentration range $(0.1$ to $100 \mu \mathrm{g} / \mathrm{L})$. Moreover, the method showed good selectivity, while the intra- and the inter-day precision indicated good reproducibility, accuracy, and precision.

The presence of EDs in foods poses health risks for humans. Particularly, cow's milk and dairy products can be sources of soluble EDs in fats because they are comparatively high in lipid fraction (Kang and Kondo, 2003). On the other hand, BPF may be detected in milk and dairy products even when the chemical nature of their packaging does not allow their release (Grumetto et al., 2013; Garcia Ibarra et al., 2019). According to the literature, in the present study, BPF levels were observed in milk samples collected in all monitored stages of milk processing. The contamination levels of raw milk from the storage tank suggest that BPF could already be present during milk production at the farm due to environmental contamination of the areas where the animals are raised (Santonicola et al., 2018). Successively, the contact with plastic materials of the dairy plant or thermic treatments during milk processing might enhance the BPF leaching in the product. BPF can be released even at room and cooling temperature, and the heating during milking can considerably facilitate its release in milk This suggests that milk pasteurization might have a role in the leaching of BPF (Shao et al., 2007; Teuten et al., 2009).

The information on the contamination levels of the BPA analogues in milk is still limited. As preliminary data, negligible contamination levels of BPF occurred in raw milk and cardboard packaged milk at the dairy company. The BPF concentrations observed in packaged milk were also below either the SML limit and t-TDI value fixed for BPA. However, the levels in milk samples were similar or, also, lower than those detected in commercial milk and dairy products (Table 2) (Liao and Kannan, 2013). The data was confirmed in a successful study in which the HPLC method above described for BPF was applied to analyze a higher number of samples collected during milk processing (Santonicola et al., 2021). A total number of 84 samples of raw milk from the storage tank, pasteurized milk from the storage tank, and cardboard packaged milk were analysed. Results showed the occurrence of BPF at concentrations (from $<\mathrm{LOQ}$ to $2.686 \mu \mathrm{g} / \mathrm{L}$ ) below the SML limit and t-TDI value fixed for BPA, and low exposure levels in different consumer age categories (Santonicola et al., 2021).

\section{Conclusions}

The developed HPLC method resulted in a simple, sensitive, and suitable analytical procedure for determining BPF levels during milk processing. The results of the preliminary application of extraction and analysis method of the milk samples collected at different stages of processing of whole milk showed the occurrence of very low levels of the analogue BPF. Particularly, raw milk and pasteurized milk from the storage tank showed relatively higher levels, probably related to the environmental contamination where the animals live and the effects of the thermic treatment at the dairy company, respectively.

Low concentrations and exposure levels of BPF were observed also when the analytical method was afterward applied to a higher number of milk samples (Santonicola et al. 2021). Nevertheless, the increasing use of BPA substitutes and the toxicological similarity between BPF and BPA represents a risk to human health because of their potential synergic ED effects on biological systems.

Better knowledge about BPF exposure

Table 2. Occurrence of BPA analogues in commercial milk and dairy products $(\mu \mathrm{g} / \mathrm{kg})$.

\begin{tabular}{lcccccccc} 
Sample & N. samples & Country & Bpa & Bpb & Bpf & Bps & References \\
Milk & 68 & Italy & $14-521$ & $16-67$ & $1-26$ & - & Grumetto et al., 2013 \\
Milk; Infant formula; Ice cream; Cheese, yogurt & 29 & Usa & $2.50 \mathrm{a}$ & $0.01 \mathrm{a}$ & $0.01 \mathrm{a}$ & $0.04 \mathrm{a}$ & Liao and kannan, 2013 \\
\hline Milk; Infant formula; Cheese, yogurt & 17 & China & $1.40 \mathrm{a}$ & $\mathrm{Nd}$ & $\mathrm{Nd}-2.30$ & Nd-0.11 & Liao and kannan, 2014 \\
\hline
\end{tabular}

ND, no detectable. a, mean value. 
levels in food is needed to assure food safety, particularly for vulnerable consumer classes. The application of monitoring systems based on the control of each stage of milk processing at the dairy company might represent a useful strategy to control the contamination of the milk chain.

\section{References}

Andújar N, Gálvez-Ontiveros Y, ZafraGómez A. Rodrigo L, Álvarez-Cubero MJ, Aguilera M, Rivas A, 2019. Bisphenol A analogues in food and their hormonal and obesogenic effects: a review. Nutrients, 11:2136.

Bansal SA, Singh AP, Kumar A, Kumar S, Kumar N, Goswamy JK, 2018. Improved mechanical performance of bisphenol-A graphene-oxide nano-composites. J Compos Mater 52:2179-88.

Caballero-Casero N, Lunar L, Rubio S, 2016. Analytical methods for the determination of mixtures of bisphenols and derivatives in human and environmental exposure sources and biological fluids. A review. Anal Chim acta 908:22-53.

Casajuana N, Lacorte S, 2004. New methodology for the determination of phthalate esters, bisphenol A, bisphenol A diglycidyl ether, and nonylphenol in commercial whole milk samples. J Agr Food Chem 52:3702-7.

Cirillo T, Latini G, Castaldi MA, Dipaola L, Fasano E, Esposito F, Cobellis L, 2015. Exposure to di-2-ethylhexyl phthalate, di-n-butyl phthalate and bisphenol A through infant formula. J Agr Food Chem 63: 3303-10.

Dragone R, Grasso G, Frazzoli C, 2020. Amperometric Cytosensor for Studying Mitochondrial Interferences Induced by Plasticizers Bisphenol B and Bisphenol A. Molecules, 25:5185.

European Food Safety Authority - EFSA (2015). Scientific opinion on the risks to public health related to the presence of bisphenol A (BPA) in foodstuffs. EFSA J, 13(1).

González N, Cunha SC, Ferreira R, Fernandes JO, Marquès $\mathrm{M}$, Nadal $\mathrm{M}$, Domingo JL, 2020. Concentrations of nine bisphenol analogues in food purchased from Catalonia (Spain): Comparison of canned and non-canned foodstuffs. Food Chem Toxicol 136:110992.

Grumetto L, Gennari O, Montesano D, Ferracane R, Ritieni A, Albrizio S, Barbato F, 2013. Determination of five bisphenols in commercial milk samples by liquid chromatography coupled to fluorescence detection. J Food Protect 76:1590-6.

Hao Z, Xiao Y, Jiang L, Bai W, Huang W, Yuan L, 2018. Simultaneous determination of bisphenol A, bisphenol F, 4nonylphenol, 4-n-nonylphenol, and octylphenol in grease-rich food by carb/PSA solid-phase extraction combined with high-performance liquid chromatography tandem mass spectrometry. Food Anal Method 11:589-97.

Ibarra VG, Sendón R, Bustos J, Losada PP, de Quirós ARB, 2019. Estimates of dietary exposure of Spanish population to packaging contaminants from cerealbased foods contained in plastic materials. Food Chem Toxicol 128:180-92.

Leepipatpiboon N, Sae-Khow O, Jayanta S, 2005. Simultaneous determination of bisphenol-A-diglycidyl ether, bisphenol-F-diglycidyl ether, and their derivatives in oil-in-water and aqueous-based canned foods by high-performance liquid chromatography with fluorescence detection. J Chromatogr A 1073:331-9.

Lestido-Cardama A, Sendón R, Bustos J, Santillana MI, Losada PP, de Quirós ARB, 2021. Multi-analyte method for the quantification of bisphenol related compounds in canned food samples and exposure assessment of the Spanish adult population. Food Packaging Shelf 28:100671.

Liao C, Kannan K, 2013. Concentrations and profiles of bisphenol $\mathrm{A}$ and other bisphenol analogues in foodstuffs from the United States and their implications for human exposure. J Agr Food Chem 61:4655-62.

Liao C, Kannan K, 2014. A survey of bisphenol A and other bisphenol analogues in foodstuffs from nine cities in China. Food Add Contaminants Part A, 31:319-29.

Mercogliano R, Santonicola S, Albrizio S, Ferrante MC, 2021. Occurrence of bisphenol A in the milk chain: A monitoring model for risk assessment at a dairy company. J Dairy Sci 104:19365.

Regulation (EU) 2018/213 of 12 February
2018 on the Use of Bisphenol A in Varnishes and Coatings Intended to Come into Contact with Food and Amending Commission Regulation (EU) No 10/2011 as Regards the Use of that Substance in Plastic Food Contact Materials.

Russo G, Barbato F, Mita DG, Grumetto L, 2019. Occurrence of Bisphenol A and its analogues in some foodstuff marketed in Europe. Food Chem Toxicol 131: 110575.

Santonicola S, Ferrante MC, di Leo G, Murru N, Anastasio A, Mercogliano R, 2018. Study on endocrine disruptors levels in raw milk from cow's farms: Risk assessment. Ital J Food Saf 7(3).

Santonicola S, Albrizio S, Ferrante MC, Mercogliano R, 2021. Study on bisphenol F, a bisphenol A analogue, at a dairy company: Health hazard and risk assessment". Food Chem Toxicol 154:112334.

Shao B, Han H, Tu X, Huang L, 2007. Analysis of alkylphenol and bisphenol A in eggs and milk by matrix solid phase dispersion extraction and liquid chromatography with tandem mass spectrometry. J Chromatogr B 850:4126.

Teuten EL, Saquing JM, Knappe DR, Barlaz MA, Jonsson S, Björn A, Takada H, 2009. Transport and release of chemicals from plastics to the environment and to wildlife. Philos T Roy Soc B 364:2027-45.

Tuzimski T, Szubartowski S, 2019. Method development for selected bisphenols analysis in sweetened condensed milk from a can and breast milk samples by HPLC-DAD and HPLC-QqQ-MS: Comparison of sorbents (Z-SEP, Z-SEP Plus, PSA, C18, Chitin and EMRLipid) for clean-up of QuEChERS extract. Molecules 24: 2093.

Usman A, Ikhlas S, Ahmad M, 2019. Occurrence, toxicity and endocrine disrupting potential of Bisphenol-B and Bisphenol-F: A mini-review. Toxicol Lett 312:222-7.

Xiao Z, Wang R, Suo D, Li T, Su X, 2020. Trace analysis of bisphenol $\mathrm{A}$ and its analogues in eggs by ultra-performance liquid chromatography-tandem mass spectrometry. Food Chem 327:126882. 